\title{
Periodic Bandwidth Allocation Based on Virtual Queue Occupancy
}

\author{
Jingdi Zeng and Nirwan Ansari \\ Advanced Networking Laboratory \\ Electrical and Computer Engineering Department \\ New Jersey Institute of Technology \\ Newark, New Jersey 07102
}

\begin{abstract}
Carrying IP traffic over connection-oriented networks requires the use of bandwidth allocation schemes at gateways or network interfaces. A new virtual queue occupancy, which is more accurate than the classical queue status parameter, is being proposed for periodic bandwidth allocation. Based on this virtual queue occupancy, an enhanced approach for lossless services, referred to as LAVQ, is simulated and evaluated in an IP/SONET environment. Simulations show that LAVQ outperforms its counterpart LAQ in terms of bandwidth utilization, without compromising the performance of queue occupancy.
\end{abstract}

\section{INTRODUCTION}

W ITH the explosive growth of Internet users, carrying IP traffic over connection-oriented networks such as ATM has been intensively studied [1-5]. Moreover, the industry [6$8]$ is envisioning the evolution of network structures that combines two technically disparate segments: the optical domain and the electrical domain. Integrating the management matured electrical domain and the bandwidth abundant optical domain is becoming a hot issue. Among a number of proposed solutions, such as IP/DWDM, IP/SONET, IP/ATM/DWDM, and IP/ATM/SONET, IP/SONET benefits from the integration simplicity and the standard maturity.

Synchronous Optical Network (SONET) was originally designed to carry voice services. Once a network allocates a circuit to a user or link, any excess bandwidth within the circuit cannot be reused. This fixed bandwidth provisioning does not utilize network resources efficiently when transferring bursty IP traffic. The resulted average circuit utilization rate of SONET is usually 5 to 10 percent in the access network and 20 to 30 percent in the core [9]. Therefore, bandwidth allocation on the network edge is a critical issue for IP/SONET, as well as for other inter-networking solutions.

IP/SONET, moreover, is characterized by other two attributes: 1. IP datagrams with different lengths make the queue behavior in IP/SONET more complicated than other solutions dealing with fixed-length cells/packets. 2. SONET allocates the bandwidth in a quantized manner. In a SONET port with the finest 1.5 Mbps granularity, for example, a 13.6 Mbps and

This work has been supported in part by OpenCon Systems, Inc., the New Jersey Commission on Higher Education via the NJI-TOWER project, and the New Jersey Commission on Science and Technology via the NJ Center for Wireless Telecommunications.

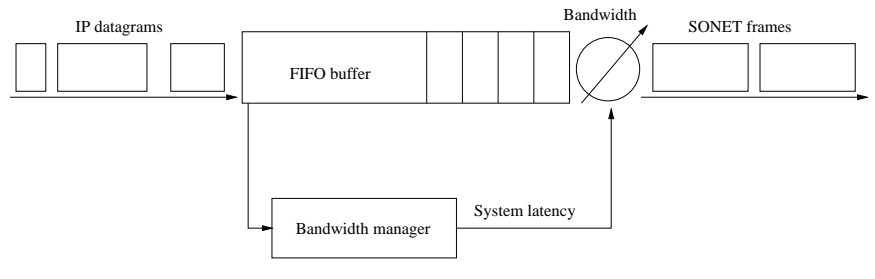

Fig. 1. Bandwidth allocation system model.

a $14.4 \mathrm{Mbps}$ bandwidth requirements all end up with a 15.0 Mbps allocation. These features infer possible modifications on current bandwidth allocation schemes in an IP/SONET environment.

In the literature, there are three categories of bandwidth allocation strategies [2]: static algorithms keep the original allocation once the allocation is set up; periodic algorithms update the bandwidth at equal time intervals; adaptive algorithms allocate the bandwidth whenever the preset conditions are met. Additionally, traffic monitoring algorithms can be classified into two broad categories: one follows the real time traffic intuitively [2$3]$; the other one learns about and predicts the incoming traffic by analyzing statistical data [4-5].

This article focuses on periodic bandwidth allocation schemes. A new virtual queue occupancy is proposed and applied to a periodic bandwidth allocation scheme tailored for IP/SONET.

The system model is described in Section II. Several periodic bandwidth allocation policies are reviewed in Section III. In Section IV, a new virtual queue occupancy for bandwidth allocation is proposed. Simulation results and performance evaluation are presented in Section V. The article concludes in Section VI.

\section{SYSTEM MODEL}

It is assumed that the traffic from a user has already been monitored by an appropriate policing mechanism, and is therefore conforming to its traffic contract with the Internet Service Provider (ISP). A simple tail-drop policy is used for packet dropping. Owing to signaling messages and other system operations, there is a system latency, a certain time lag between the time the required bandwidth is calculated and the time the update is actually reflected in the capacity of the connection. 


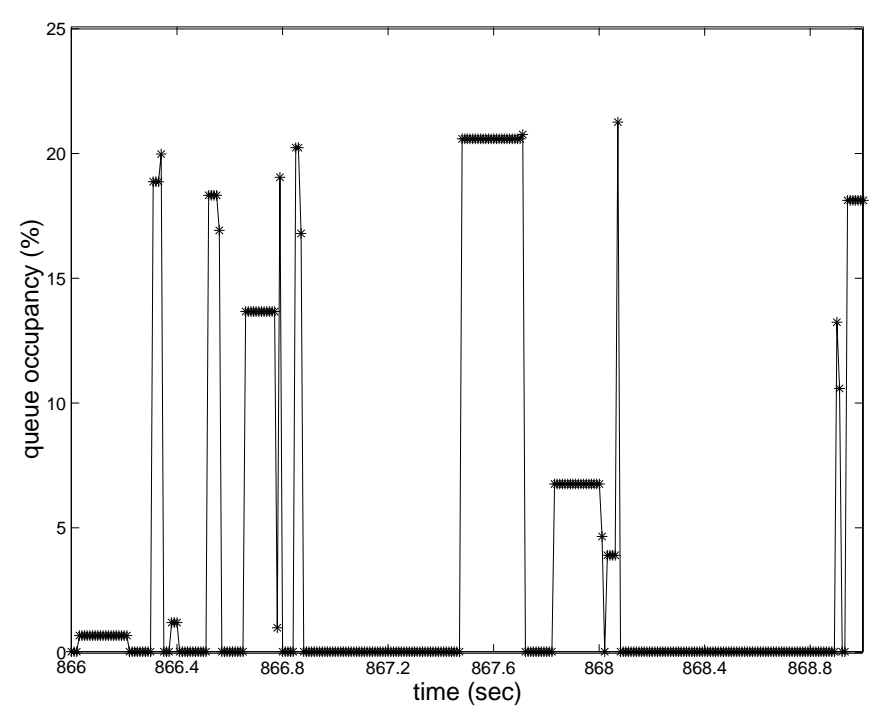

Fig. 2. Queue occupancy (LAQ) vs. time.

Accordingly, a user or link is modeled as a single server queue utilizing a bandwidth manager with certain system latency, as shown in Fig. 1.

The following notation is used throughout this article:

$\Delta T$ - the adjustment period, which is the update frequency of bandwidth allocation.

$\Delta t$ - the sampling slot, which is the sampling frequency of arrival rates.

$\Delta d$ - the system latency, which is the length of time necessary for the update to be effective.

$\Delta O$ - the buffer idle timer, which records how long the buffer has been empty in an adjustment period $\Delta T$.

$\Delta G$ - the finest SONET granularity, which is assumed to be 1.5 Mbps.

$\lambda_{i}$ - the average arrival rate in the adjustment period $i$.

$R_{p}$ - the peak arrival rate.

$q_{i}$ - the queue occupancy at the end of period $i$.

$Q_{i}$ - the virtual queue occupancy at the end of period $i$, which is drawn from $q_{i}$ and will be described in depth in Section IV.

$c_{i}$ - the required bandwidth estimated for period $i$.

$C_{i}$ - the actually allocated bandwidth for period $i$, due to SONET granularity, i.e., $\left\lceil\frac{c_{i}}{\Delta G}\right\rceil \times \Delta G$.

$\rho$ - bandwidth utilization, i.e., $\frac{\sum \lambda_{i}}{\sum C_{i}}$, which is affected by

SONET granularity, and is thus called the granular utilization in the rest of the article.

$B$ - the preset buffer size.

\section{PERIODIC BANDWIDTH ALLOCATION SCHEMES}

While static and adaptive bandwidth on demand schemes have the best complexity and flexibility performance, respectively, periodic schemes make a better compromise because they allow acceptable complexity and flexibility at the same time. A periodic scheme calculates and updates the demanded bandwidth for one period by using parameters derived from the last period, such as queue length values and arrival rates.

Using the average arrival rate, a basic policy is to allocate for the next period the amount of bandwidth required to transmit the same amount of traffic that has arrived in the previous period [2]. Thus the allocated bandwidth for period $i$ is determined from

$$
c_{i}=\lambda_{i-1}
$$

However, when traffic increases, the allocation may fall short of the requirement; when traffic decreases, this scheme may waste resources by still allocating the same amount of bandwidth as before. As a result, there is tremendous research work undergoing on the IP traffic prediction, which is not the focus of this article though.

An alternative strategy [3] multiplies the assigned bandwidth by a constant factor $\mathrm{C}$, which is greater than 1 . Thus the allocated bandwidth for period $i$ follows

$$
c_{i}=C \times \lambda_{i-1}
$$

Under the same circumstances, this strategy has shorter queue and smaller loss than those of the previous one, depending on how aggressively the factor $\mathrm{C}$ is scaled. The dumb parameter $\mathrm{C}$, however, introduces uncertainty to the system.

Instead of dealing with the average arrival rate, some other schemes [2-3] employ the queue occupancy up to the beginning of the current period. They allocate for period $i$ the amount of bandwidth needed to drain what has accumulated in the queue during the previous period $i-1$, which is

$$
c_{i}=\frac{q_{i-1}}{\Delta T} .
$$

The queue status preciseness, as the only parameter, however, may not be robust enough.

One comprehensive policy [2], referred to as Last Arrival plus Queue occupancy (LAQ) in this article, takes both the arrival rate and queue status into consideration. It allocates for the next period the amount of bandwidth necessary to transmit the current content of the queue plus the same amount of traffic that has arrived in the previous period. Therefore, the allocated bandwidth for period $i$ becomes

$$
c_{i}=\lambda_{i-1}+\frac{q_{i-1}}{\Delta T}
$$

Comparing to strategies with prediction ability, this is a simple but still effective method, without requiring any knowledge beyond what is currently known. For IP/SONET, however, this bandwidth allocation scheme has its drawbacks and thus needs to be enhanced, as will be discussed in Section IV.

\section{VIRTUAL QUEUE OCCUPANCY}

LAQ [2-3], one of the few periodic bandwidth allocation schemes with better performance, measures the average arrival rate $\lambda_{i-1}$ in period $i-1$, determines the queue occupancy $q_{i-1}$ 
at the end of period $i-1$, and then allocates bandwidth for period $i$ as follows:

$$
c_{i}= \begin{cases}\lambda_{i-1} & q_{i-1}=0 \\ \lambda_{i-1}+\frac{q_{i-1}}{\Delta T} & 0<q_{i-1}<B \\ \lambda_{i-1}+\frac{B}{\Delta T} & q_{i-1}=B\end{cases}
$$

where B is the preset buffer size.

The performance of LAQ depends on how accurate the average arrival rate and the queue occupancy are. Since LAQ has no prediction ability, the smaller sampling slots and adjustment periods are, the better average arrival rates follow the traffic trend, and the better queue occupancy values reflect the queue status change. Regarding to these two types of parameters for bandwidth allocation strategies, the queue status preciseness has not gained as much attention as that of the traffic measurement and prediction.

Obviously, the average data arrival to the buffer may not be equal to the bandwidth assigned to this buffer. As a result, overflows or underflows will likely happen if the allocated bandwidth is not adjusted properly. In general, buffer overflows occur when the assigned bandwidth is less than the average data arrival, and buffer underflows occur when the allocated bandwidth is more than the average data arrival. In the extreme case, the buffer is empty. When overflows or underflows happen, however, queue occupancy $q_{i-1}$, an instantaneous value, does not always reflect the real buffer status because the bandwidth manager does not know whether the buffer status is newly changed or has lasted for a while.

Next, two bandwidth allocation scenarios shall be presented, regarding the impact resulted from the queue status.

Scenario 1: a buffer is newly empty at the end of period $i$, which definitely infers a slow arrival rate, thus the bandwidth manager should reduce the bandwidth allocation in the upcoming period. According to (5), when the queue occupancy $q_{i}$ becomes 0 , the allocated bandwidth for period $i$ does reduce to (1).

Scenario 2: a buffer has been empty for a while, which indicates that the allocated bandwidth has been more than necessary and the bandwidth manager should have reduced bandwidth allocation more aggressively. Therefore now the bandwidth manager needs to allocate less bandwidth than that of the scenario 1. According to (5), however, the queue occupancy $q_{i}$ is 0 , and the allocated bandwidth is still the same as (1).

One way to counteract this effect is to extend the queue occupancy $q_{i}$ beyond its range $[0, B]$ so that it reflects the buffer status more precisely. The extended value, referred to as virtual queue occupancy $Q_{i}$, is yielded based on a buffer idle timer $\Delta O$ that captures the time duration in which the buffer has been empty during an adjustment period $\Delta T$. Furthermore, only the latest duration where the buffer remains empty up to the end of the adjustment period is considered. Thus, the modified bandwidth allocation for period $i$ becomes

$$
c_{i}=\lambda_{i-1}+\frac{Q_{i-1}}{\Delta T},
$$

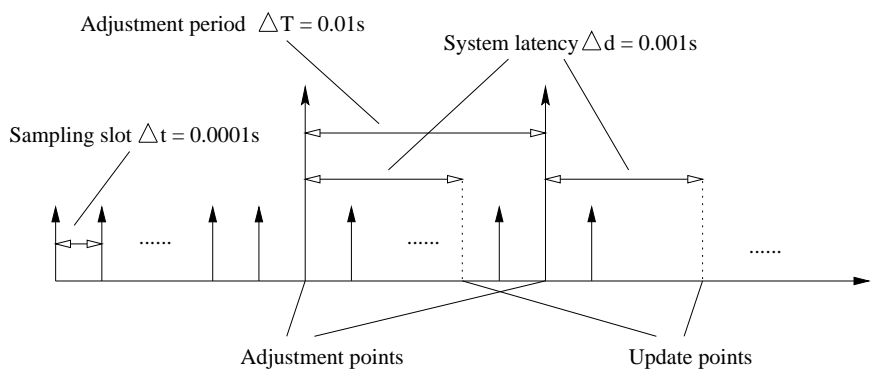

Fig. 3. Periodic measurement.

where

$$
Q_{i-1}=q_{i-1}-\Delta O \times C_{i-1} .
$$

When the buffer is empty, queue occupancy $q_{i-1}$ becomes 0 , and thus the virtual queue occupancy turns out as

$$
Q_{i-1}=-\Delta O \times C_{i-1} \text {. }
$$

With the extended range $\left[-\Delta O \times C_{i}, B\right]$, the virtual queue occupancy $Q_{i}$ minimizes the inaccurancy due to a prolonged empty buffer. Numerically, it reduces the bandwidth requirement by $\frac{\Delta O}{\Delta T} \times C_{i-1}$ when the buffer has been empty for the duration of $\Delta O$, which is rather frequent in an IP/SONET environment.

As shown in Fig. 2, owing to SONET bandwidth granularity, the buffer is empty quite often when using LAQ for bandwidth allocation. This phenomenon affects queue occupancy preciseness, and therefore bandwidth allocation preciseness, as explained in Scenario 2. The performance of bandwidth allocation schemes using classical queue occupancy and virtual queue occupancy, respectively, will be simulated and compared in Section V.

\section{Simulation Results}

As shown in Fig. 3, the bandwidth manager samples related parameters every $\Delta t$ seconds and calculates the bandwidth allocation every $\Delta T$ seconds. The updated bandwidth allocation takes place after the system latency $\Delta d$. To make this periodic calculation meaningful, the bandwidth manager should complete updating bandwidth allocation before the next round of calculation, i.e., $\Delta d \leq \Delta T$.

According to the industry implementation [9], which states that the dynamic allocation on a SONET node can be achieved in one millisecond, it is assumed that the system latency $\Delta d=$ 0.001 seconds. Moreover, the bandwidth manager is assumed to adjust the bandwidth every $\Delta T=10 \Delta d=0.01$ seconds, providing enough time for the bandwidth to be updated. Additionally, it is assumed that the bandwidth manager measures arrival rates every $\Delta t=0.0001$ seconds to capture the traffic arrival characteristic as precisely as possible. The average arrival rate $\lambda_{i}$ and the granular utilization $\rho$ are collected every 100 sampling slots and 100 adjustment periods, respectively. 


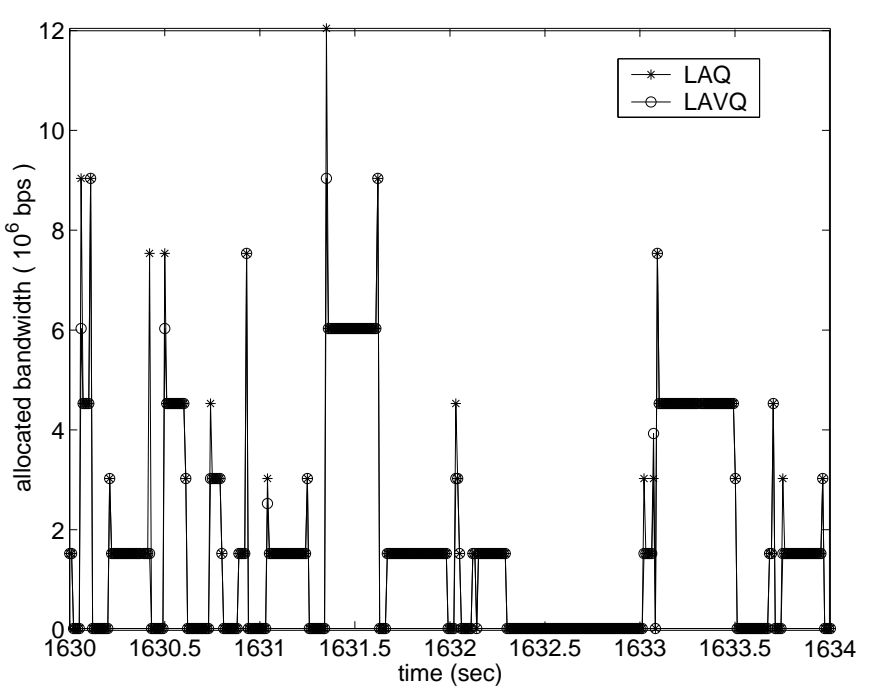

Fig. 4. Bandwidth allocation (LAQ, LAVQ) vs. time.

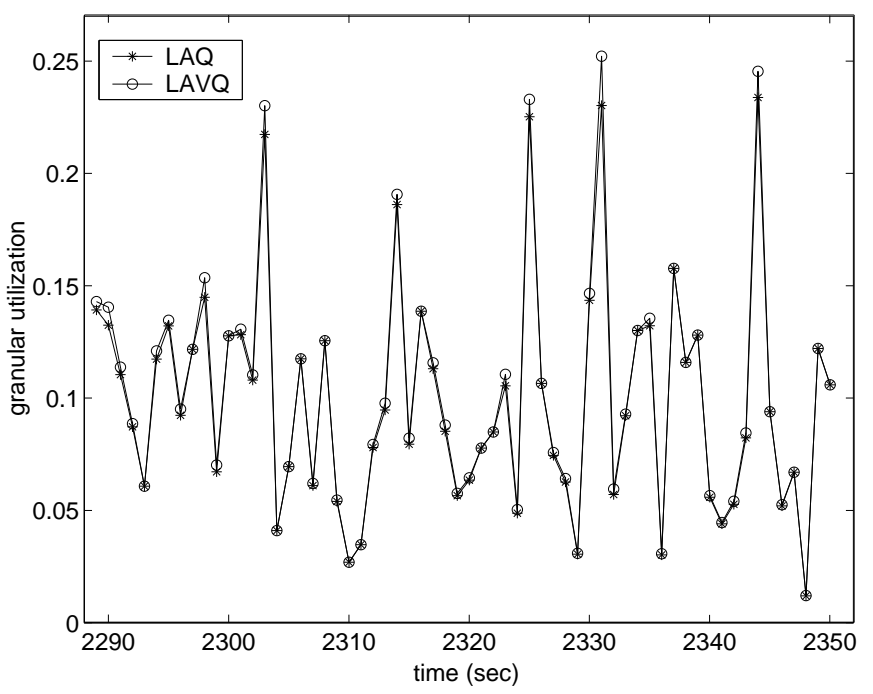

Fig. 5. Granular utilization $\rho$ (LAQ, LAVQ) vs. time.

The following simulations are based on these system assumptions and parameters.

The performance of bandwidth allocation schemes is simulated in a First In First Out (FIFO) buffer ( $B=130 \mathrm{~K}$ bits $)$, which provides lossless services (the derivation of the required buffer size for lossless services is omitted because of the limited space). A couple of resources with exponentially distributed session holding and inter-arrival time [10], respectively, are aggregated. The packet inter-arrival time is Pareto distributed, while the size of the packets is generated following an exponential distribution with a mean of 1500 bytes. Each simulation lasts for a 40-minute period, corresponding to 240 thousand adjustment periods and 24 million sampling slots.

By replacing the queue occupancy $q_{i}$ with the virtual queue

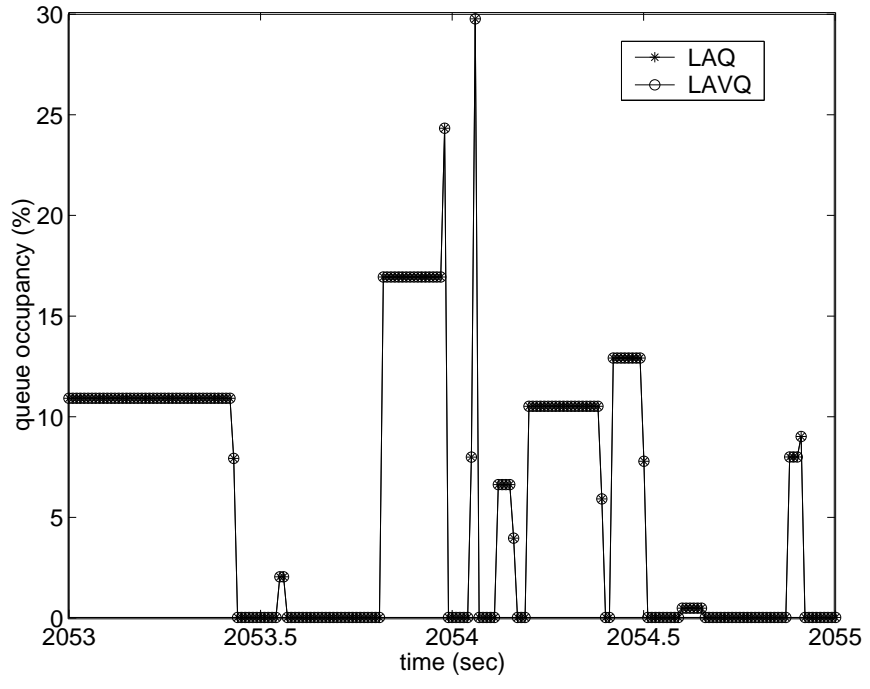

Fig. 6. Queue occupancy (LAQ, LAVQ) vs. time.

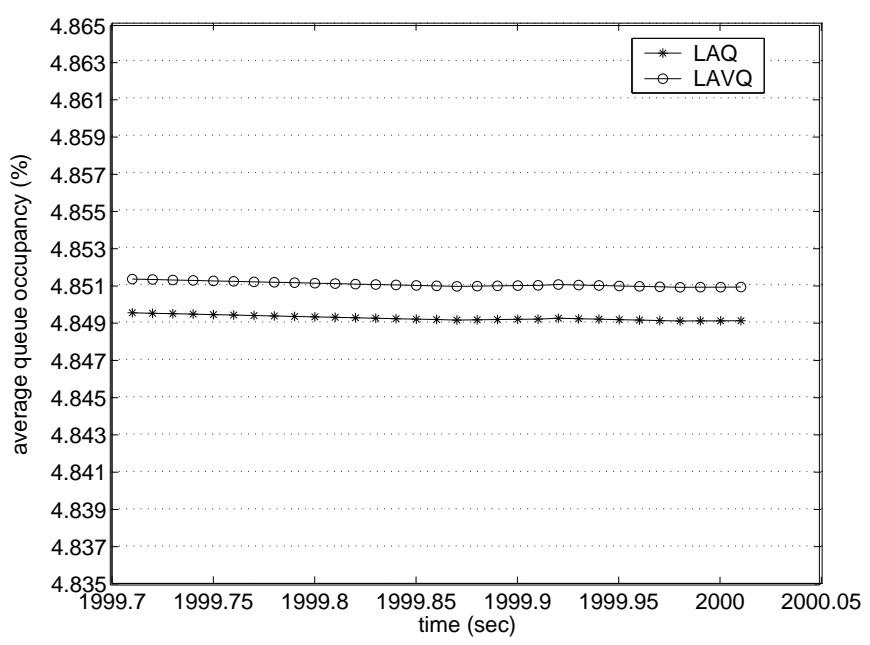

Fig. 7. Average queue occupancy (LAQ, LAVQ) vs. time.

occupancy $Q_{i}$, an enhanced bandwidth allocation scheme, referred to as Last Arrival and Virtual Queue occupancy (LAVQ), allocates less bandwidth than what LAQ does, as shown in Fig. 4 in a small time scale. Maintaining at least the same performance of LAQ, LAVQ regains over-assigned bandwidth when the buffer underflows. The accumulated improvement is illustrated in Fig. 5, where LAVQ achieves bigger granular utilization $\rho$ and thus better bandwidth utilization. To assure that the buffer space is not traded for the performance improvement on bandwidth utilization, the queue occupancy of LAQ and LAVQ is compared in Fig. 6 and in Fig. 7, respectively. Simulations show that LAQ and LAVQ have very slight difference in term of queue occupancy.

Another issue which is worth mentioning is the system latency. To provide lossless services, LAVQ needs a buffer to 
hold the underestimated traffic and the delayed traffic resulted from the system latency $\Delta d$. From simulation results which are not included in this article, it is observed that the ratio of system latency and adjustment period, $\frac{\Delta d}{\Delta T}$, has a big impact on the queue status. When the system latency increases, the queue occupancy grows in a nonlinear pattern. The system latency, $\Delta d$, however, changes unpredictably because it is related to other unforeseeable factors, such as traffic loads and available network resources. This uncertainty induces the unexpected queue occupancy influctuation, which in turn may cause extra delay jitter.

\section{CONCLUSIONS}

This article has proposed a new virtual queue occupancy which indicates a more accurate queue status for bandwidth allocation. An enhanced periodic bandwidth allocation scheme, LAVQ, has subsequently been proposed based on this new measure. LAVQ achieves better bandwidth utilization than its counterpart LAQ, with a negligible requirement for extra buffer resources. Further work is considered on the overflow duration defined by different thresholds, where IP datagram dropping strategies may play important roles. In addition, using the traffic prediction to strengthen the preciseness of average arrival rates will certainly improve the accuracy of bandwidth allocation.

\section{REFERENCES}

[1] A. L. B. P. B. Diniz, C. C. Goulart, and J. M. S. Nogueira, "A dynamic bandwidth allocation service for ATM-based networks supporting multimedia applications", in Proc. 1st IEEE Int. Conf. ATM (ICATM'98), Colmar, France, pp. 292-299, Jun. 1998.

[2] Y. Afek, M. Cohen, E. Haalman, and Y. Mansour, "Dynamic bandwidth allocation policies", in Proc. IEEE INFOCOM'96, San Francisco, U.S.A., pp.880-887, Mar. 1996.

[3] J. Brassil, "Overcoming reservation latency for IP traffic in an ATM PON", in Proc. IEEE GLOBECOM'99, Rio de Janeiro, Brazil, pp.11551161, Dec. 1999.

[4] Q. K. Mehra, B. Ravichandran, J. B. D. Cabrera, D. N. Greve, and R. S. Sutton, "Towards self-learning adaptive scheduling for ATM networks", in Proc. 36th Conf. Decision \& Control, San Diego, U.S.A., pp.23922398, Dec. 1997.

[5] F. Zhao, M. Hanawa, and M. Takahara, "Traffic measurement-based admission control Using Rate Envelope in ATM Networks", in Proc. IEEE Int. Conf. Networks (ICON’00), Singapore, pp.223-229, Sept. 2000.

[6] A. Copley, "Optical domain service interconnect (ODSI): defining mechanisms for enabling on-demand high-speed capacity from the optical domain", IEEE Commun. Mag., vol.38, no.10, pp.168-174, October 2000.

[7] J. Manchester, J. Anderson, B. Doshi, and S. Dravida, "IP over SONET", IEEE Commun. Mag., vol.36, no.5, pp.136 -142, May 1998.

[8] R. Jain, "IP over SONET", March 1998. [Online]. Available: www.cis.ohio-state.edu/ $/$ jain/talks/ipsonet.htm.

[9] G. Lee, "FlexBand technology optimizes SONET", White Paper, Geyser Networks, Sunnyvale, U.S.A., 2001.

[10] J. Färber, S. Bodamer, J. Charzinski, "Measurement and Modeling of Internet Traffic at Access Networks", in Proc. EUNICE '98 Open Eur. Summer School on Network Management and Operation, München, Germany, pp. 196-203, Aug. 1998. 